\title{
Nghiên cứu các nhân tố ảnh hưởng đến kết quả hoạt động xuất khẩu của các doanh nghiệp thủy hải sản vùng Đồng bằng sông Cửu Long
}

\section{Studying factors affecting the export performance of seafood enterprises in the Mekong Delta.}

\author{
Nguyễn Thị Lệ ${ }^{1 *}$, Huỳnh Thanh Nhã², Nguyễn Thiện Phong ${ }^{3}$ \\ ${ }^{1}$ Trường Cao đẳng Kinh tế - Kỹ thuật Cần Thơ, Việt Nam \\ ${ }^{2}$ Trường Đại học Kỹ thuật - Công nghệ Thành phố Cần Thơ, Việt Nam \\ ${ }^{3}$ Trường Đại học Tây Đô, Việt Nam \\ "Tác giả liên hệ, Email: ntle@ctec.edu.vn
}

\begin{abstract}
THÔNG TIN
TÓM TẮT

DOI: $10.46223 / \mathrm{HCMCOUJS}$.

soci.vi.15.1.604.2020

Ngày nhận: 27/12/2019

Ngày nhận lại: 31/01/2020

Duyệt đăng: 07/07/2020

Tù khóa:

doanh nghiệp thủy hải sản, kết quả hoạt động xuất khẩu, nhân tố ảnh hưởng

Nghiên cứu được thực hiện hướng đến mục tiêu phân tích các nhân tố ảnh hưởng đến kết quả hoạt động xuất khẩu của các doanh nghiệp thủy hải sản vùng Đồng bằng sông Cửu Long. Dữ liệu sử dụng trong nghiên cứu được thu thập bằng phương pháp chọn mẫu thuận tiện với cỡ mẫu là 170 doanh nghiệp có hoạt động xuất khẩu thủy hải sản trong vùng. Kết quả của kiểm định thang đo Cronbach's Alpha, phân tích nhân tố khám phá EFA và phân tích hồi qui tuyến tính cho thấy có bốn nhóm nhân tố ảnh hưởng đến kết quả hoạt động xuất khẩu của các doanh nghiệp thủy hải sản trong vùng bao gồm năng lực cạnh tranh của doanh nghiệp, đặc điểm và khả năng của doanh nghiệp, yếu tố quan hệ của doanh nghiệp và khả năng xây dựng thương hiệu của doanh nghiệp. Kết quả của nghiên cứu là căn cứ đề xuất các khuyến nghị góp phần nâng cao kết quả hoạt động xuất khẩu của các doanh nghiệp thủy hải sản ở vùng Đồng bằng sông Cửu Long.
\end{abstract}

Keywords:

seafood enterprises, export performance, influencing factors

\section{ABSTRACT}

The study aimed to analyze factors affecting the export performance of seafood enterprises in the Mekong Delta. The data of the study were collected from a sample of 170 enterprises involved in seafood export in the region. The results of Cronbach's Alpha, factor analysis EFA and linear regression analyses show that there are four groups of factors affecting the export performance of seafood enterprises including core competencies, reputation and capabilities, relationships and brand image. The results of the study are the basis for proposing 
recommendations to improve the export performance of seafood businesses in the Mekong Delta.

\section{Giới thiệu}

Hiện nay thủy hải sản vẫn được coi là mặt hàng xuất khẩu chủ lực của Việt Nam. Theo Phòng Thương mại và Công nghiệp Việt Nam (2017), Việt Nam đứng trong ba quốc gia hàng đầu thế giới (sau Trung Quốc và Ân Độ) về nuôi trồng và xuất khẩu thủy hải sản. Tuy nhiên, các vấn đề về bệnh dịch, hạn hán, xâm nhập mặn hay ô nhiễm môi trường tại Việt Nam hiện đang tạo ra nhiều khó khăn và áp lực cho sự phát triển của ngành. Nhìn từ nghiên cứu của Phan (2009) cho thấy việc sản xuất và tiêu thụ thủy hải sản của các doanh nghiệp (DN) Việt Nam còn nhiều thiếu sót và sự không nhất quán. Điều đó trực tiếp làm ảnh hưởng đến hiệu quả hoạt động và tính bền vững trong sản xuất, tiêu thụ và tiếp thị sản phẩm. Hệ thống cơ sở hạ tầng đã không đáp ứng yêu cầu thực tế, các cơ sở sản xuất nhỏ lẻ, rải rác, các mô hình công nghệ cao còn hạn chế, mối liên kết trong sản xuất và quản lý còn lỏng lẻo. Bên cạnh đó, giá thủy hải sản nguyên liệu không hợp lý so với giá xuất khẩu do chi phí sản xuất trong nước cao (Xuan Thao, 2017) cũng là một trong các yếu tố tạo nên khó khăn lớn để các DN Việt Nam có thể cạnh tranh trên thị trường xuất khẩu.

Để phát triển bền vững trên thị trường quốc tế, các $\mathrm{DN}$ thủy hải sản chú trọng vào việc nâng cao hoạt động xuất khẩu. Tuy nhiên các rào cản kỹ thuật từ các quốc gia nhập khẩu ngày càng nhiều hơn và khắt khe hơn đã đẩy các $\mathrm{DN}$ thủy hải sản vào tình thế khó khăn hơn. Để đạt được kết quả tốt trong hoạt động xuất khẩu đòi hỏi họ phải có chiến lược thông minh và lâu dài hơn, tìm kiếm được thị trường ổn định và phù hợp hơn. Ở các thị trường quốc tế, nơi cạnh tranh ngày càng tăng, yêu cầu cho sự thành công của $\mathrm{DN}$ nằm ở việc xác định các yếu tố ảnh hưởng đến hoạt động xuất khẩu và giải pháp hành động có liên quan. Để vượt qua các rào cản từ bên ngoài, $\mathrm{DN}$ cần trang bị cho mình các nguồn lực bên trong đủ mạnh nhằm giúp $\mathrm{DN}$ thích ứng tốt với các thay đổi từ môi trường, đồng thời ứng phó với các đối thủ cạnh tranh trong và ngoài nước. Tuy nhiên, để gọi tên chính xác các nguồn lực này là gì thì còn khá ít các nghiên cứu đã được thực hiện, đặc biệt là trong ngành thủy hải sản của vùng Đồng bằng sông Cửu Long (ĐBSCL) nói riêng và Việt Nam nói chung.

Trong địa bàn nghiên cứu là vùng ĐBSCL hiện tại có hai nghiên cứu đã được công bố về kết quả hoạt động xuất khẩu thủy hải sản của các $\mathrm{DN}$ là của $\mathrm{V}$. B. Nguyen, Le, Nguyen, và Vo (2017) và T. V. Nguyen, Phuoc, Nguyen, Phan, và Vo (2018). Cả hai nghiên cứu này thực tế sử dụng cùng một mô hình nền tảng bởi cùng nghiên cứu các yếu tố ảnh hưởng đến hiệu suất xuất khẩu trên quan điểm xem xét cả yếu tố bên trong $\mathrm{DN}$ và bên ngoài $\mathrm{DN}$. Nhằm hướng đến việc định hướng cho những điều chỉnh từ các nguồn lực hay các hoạt động bên trong DN, nên "Nghiên cứu các nhân tố ảnh hưởng đến kết quả hoạt động xuất khẩu của các doanh nghiệp thủy hải sản vùng Đồng bằng sông Củ̉u Long” là cấp thiết, cần được thực hiện để có cơ sở, làm căn cứ đề xuất các khuyến nghị cho $\mathrm{DN}$. Nghiên cứu này được thực hiện trong phạm vi giới hạn các yếu tố nguồn lực bên trong của $\mathrm{DN}$ mà không bao quát cả bên trong và bên ngoài như hai nghiên cứu đã công bố kể trên. 


\section{Cơ sở lý thuyết và mô hình đề xuất}

Với sự tăng trưởng nhanh chóng của hoạt động kinh doanh quốc tế và quốc tế hóa, xuất khẩu đóng một vai trò quan trọng quyết định sự sống còn và tăng trưởng của $\mathrm{DN}$ (Chen, Sousa, $\& \mathrm{He}, 2016)$. Theo Adu-Gyamfi và Korneliussen (2013) để duy trì tính cạnh tranh trong thị trường toàn cầu thì nhất thiết phải cải thiện kết quả của hoạt động xuất khẩu. Xuất khẩu là một chiến lược cơ bản để đảm bảo sự tồn tại và tăng trưởng của $\mathrm{DN}$, việc các $\mathrm{DN}$ có thể đạt được lợi thế cạnh tranh trong thị trường quốc tế có ảnh hưởng tích cực đến hoạt động xuất khẩu hiện tại và tương lai (Navarro, Losada, Ruzo, \& Diez, 2010). Nghiên cứu này cũng cho rằng kết quả hoạt động xuất khẩu là mức độ mà công ty hoàn thành mục tiêu của mình khi bán một mặt hàng trên thị trường thế giới. Altıntaş, Tokol, và Harcar (2007) tin rằng kết quả hoạt động xuất khẩu cũng thể hiện thông qua mức độ hài lòng với hoạt động xuất khẩu mà DN đã thực hiện.

Trên cơ sở các nghiên cứu trên, nghiên cứu này tiếp cận khái niệm kết quả hoạt động xuất khẩu theo quan điểm của quản trị năng lực (Competence-based View-CBV), tức tập trung vào khả năng sử dụng, kết hợp tài sản, nguồn, năng lực nhằm đạt được tăng trưởng và hiệu quả tổng thể của tổ chức. Lý thuyết $\mathrm{CBV}$ đã được phát triển bởi khá nhiều nghiên cứu nhưng nổi bật nhất là của Sanchez và Heene (1996). Lý thuyết này cho rằng năng lực là khả năng duy trì, triển khai và phối hợp các nguồn lực và khả năng theo cách giúp công ty đạt được mục tiêu. Hay nói cách khác, năng lực của công ty sẽ quyết định sự thành công của công ty đó. Lý thuyết CBV giả định:

(1) Công ty thành công chính là mức độ đạt được và thỏa mãn liên tục những mục tiêu của công ty;

(2) Công ty thành công $=\mathrm{f}$ (các nguồn lực, các khả năng, quá trình quản trị, chiến lược phù hợp).

Căn cứ trên giả định này nghiên cứu xây dựng biến đo lường sự thành công của công ty chính là kết quả hoạt động xuất khẩu dựa trên đánh giá của mỗi DN theo mức độ đạt được và hài lòng với mục tiêu của $\mathrm{DN}$. Để tạo ra được kết quả xuất khẩu này là sự tác động từ các yếu tố xuất phát từ bên trong $\mathrm{DN}$ bao gồm các nguồn lực, khả năng, kể cả năng lực quản lý hay khả năng thực hiện các chiến lược.

Nghiên cứu của V. B. Nguyen và cộng sự (2017) đã chứng minh được đặc điểm và khả năng của $\mathrm{DN}$, năng lực quản lý, và chiến lược marketing xuất khẩu tác động đến kết quả xuất khẩu của DN trong ngành thủy hải sản. Trong đó chiến lược marketing xuất khẩu được xem xét như một biến trung gian tác động đến kết quả xuất khẩu. Tuy nhiên hai yếu tố còn lại ngoài việc tác động đến chiến lược marketing xuất khẩu thì cũng được chứng minh có tác động trực tiếp lên kết quả xuất khẩu của DN. Nghiên cứu của T. V. Nguyen và cộng sự (2018) cũng chứng minh điều tương tự. Điểm mới của nghiên cứu này so với $\mathrm{V}$. B. Nguyen và cộng sự (2017) là đưa thêm yếu tố mối quan hệ vào mô hình, tuy nhiên, chưa chứng minh được yếu tố này có tác động đến kết quả xuất khẩu. Các yếu tố liệt kê của hai nghiên cứu trên là nhóm các yếu tố bên trong và phù hợp với quan điểm quản trị năng lực $\mathrm{CBV}$, nhưng cả hai nghiên cứu này đã không nghiên cứu tách rời nhóm các yếu tố bên trong với bên ngoài mà đã chứng minh được khá nhiều các yếu tố như đặc điểm ngành, đặc điểm thị trường xuất khẩu, đặc điểm thị trường nội địa tác động đến hiệu suất xuất khẩu. Vận dụng lý thuyết $\mathrm{CBV}$ và kế thừa kết quả của hai nghiên cứu trên nghiên cứu này sẽ xây dựng các nhân tố thuộc về nguồn lực hay khả năng bên trong của DN thành các biến độc lập để xem xét mối quan hệ với kết quả xuất khẩu. Các nguồn lực này 
được mở rộng ra trên nhiều nhân tố bao gồm cả khả năng cạnh tranh và khả năng xây dựng thương hiệu.

Theo quan điểm của Porter (2008), cạnh tranh là vấn đề cơ bản quyết định sự thành công hay thất bại của $\mathrm{DN}$. Chiến lược cạnh tranh là sự tìm kiếm vị thế cạnh tranh thuận lợi trong ngành. Vị thế cạnh tranh khi xem xét trên phương diện từ $\mathrm{DN}$ thì đó chính là khả năng cạnh tranh của $\mathrm{DN}$. Guan, Yam, Mok, và Ma (2006) tin rằng khả năng cạnh tranh bao gồm nhiều yếu tố như lãnh đạo, chi phí, lợi thế giá trị khách hàng, quản lý thời gian hiệu quả, và lợi thế của sự đổi mới. Một công ty có lợi thế cạnh tranh nếu nó có thể sản xuất và bán sản phẩm với giá thấp hơn đối thủ cạnh tranh (Rojoka, 2009). Năng lực cạnh tranh bao gồm các khả năng cho phép tổ chức để phân biệt chính nó với các đối thủ cạnh tranh và năng lực cạnh tranh có được thông qua việc ra quyết định của nhà quản lý. Hai trong số các năng lực cạnh tranh có thể được sở hữu bởi một công ty là chi phí thấp và sự khác biệt. Mặt khác, Salloum (2013) đã kết luận rằng các môi trường kinh doanh khác nhau sẽ làm thay đổi các nhân tố dùng để đo lường năng lực cạnh tranh của DN. Karim, Smith, Halgamuge, và Islam (2008) đã so sánh và chứng minh các đo lường cho năng lực cạnh tranh ở các quốc gia khác nhau thì không hoàn toàn giống nhau.

Nếu như các nghiên cứu trên là để tìm hiểu về năng lực cạnh tranh trên nhiều khía cạnh khác nhau từ các cách thức đo lường, cho đến các quan điểm đo lường trên từng quốc gia khác nhau thì Han, Chen, và Ebrahimpour (2007) lại tập trung vào nghiên cứu các mối quan hệ nhân quả của năng lực cạnh tranh và cho rằng hiệu quả kinh doanh sẽ tăng nếu công ty có năng lực cạnh tranh mạnh hơn thông qua cải tiến các nhân tố có thể tác động đến năng lực này. Đến năm 2017 nghiên cứu của Famiyeh cũng tiếp tục chứng minh mối quan hệ của năng lực cạnh tranh và kết quả kinh doanh. Tuy nhiên những nghiên cứu chuyên sâu theo hướng này còn khá hạn chế trong ngành xuất khẩu thủy hải sản. Đây chính là cơ sở hình thành giả thuyết cho nghiên cứu này với $H 1$ : năng lục cạnh tranh tác động tích cực đến kết quả hoạt động xuất khẩu của $D N$ thủy hải sản vùng ĐBSCL.

Nghiên cứu của Chen và cộng sự (2016) chứng minh rằng các đặc điểm và khả năng của $\mathrm{DN}$ là nhân tố rất quan trọng ảnh hưởng đến kết quả hoạt động xuất khẩu cũng như sự cạnh tranh của các công ty. Các đặc điểm này bao gồm quy mô của công ty, kinh nghiệm xuất khẩu, năng lực quốc tế, số năm thành lập, và kiến thức thị trường xuất khẩu (Nazar \& Saleem, 2009). Chen và cộng sự (2016) đã bổ sung thêm việc lập kế hoạch xuất khẩu và định hướng thị trường xuất khẩu cũng là hai yếu tố quan trọng hình thành đặc điểm và khả năng của mỗi $\mathrm{DN}$. Đồng tình với quan điểm trên, nghiên cứu này đề xuất giả thuyết $H 2$ : đặc điểm và khả năng của $D N$ tác động cùng chiều đến kết quả hoạt động xuất khẩu của DN thủy hải sản vùng ĐBSCL.

Bộ phận quản lý trong bất cứ ngành nghề nào cũng giữ vai trò định hướng và kiểm soát hoạt động của $\mathrm{DN}$, do đó, các yếu tố quản lý thực hiện nhiệm vụ then chốt đối với thành công trong hoạt động kinh doanh (Chen et al., 2016). Quản lý trong lĩnh vực xuất khẩu là đưa ra quyết định và các chiến lược để tăng cường và mở rộng thị trường nước ngoài, và chắc chắn sẽ ảnh hưởng đến kết quả hoạt động xuất khẩu của DN (Katsikeas, Leonidou, \& Morgan, 2000). Trong năng lực quản lý thì các yếu tố như thái độ, kinh nghiệm hay trình độ của người ra quyết định luôn được đề cao. Hơn thế, Chen và cộng sự (2016) tiết lộ rằng các nhà quản lý với kinh nghiệm quốc tế là yếu tố chính quyết định hiệu quả hoạt động xuất khẩu. Nghiên cứu của Moghaddam, Hamid, và Aliakbar (2012), Chen và cộng sự (2016) và V. B. Nguyen và cộng sự (2017) cũng đã chỉ ra rằng các yếu tố năng lực quản lý có tác động đến hoạt động xuất khẩu. 
Do đó, giả thuyết H3: Năng lục quản lý tác động cùng chiều đến kết quả hoạt động xuất khẩu của $D N$ thủy hải sản vùng $Đ B S C L$ được đề xuất trong nghiên cứu này.

Các nghiên cứu của Agus, Isa, Farid, và Permono (2015), Chen và cộng sự (2016), Erdil và Ozemir (2016), Arafah, Batara, và Hady (2018) cho thấy các DN áp dụng chiến lược marketing xuất khẩu đã tạo ra tác động tốt đến kết quả hoạt động xuất khẩu. Chiến lược marketing xuất khẩu được xem là công cụ mà một $\mathrm{DN}$ sử dụng để phản ứng với các thay đổi của thị trường và để đáp ứng các mục tiêu xuất khẩu (Moghaddam et al., 2012). Nó liên quan đến tất cả các khía cạnh của một kế hoạch marketing thông thường bao gồm chiến lược sản phẩm, giá cả, khuyến mãi và phân phối (Chen et al., 2016). Các nghiên cứu trên được thực hiện cho hoạt động xuất khẩu trong nhiều ngành nghề mà ít có nghiên cứu chứng minh được mối quan hệ này trong hoạt động xuất khẩu thủy hải sản ngoại trừ nghiên cứu của $\mathrm{V}$. $\mathrm{B}$. Nguyen và cộng sự (2017). Từ các dẫn chứng trên, giả thuyết $H 4$ : được thực hiện để kiểm chứng tác động tích cực của chiến lược marketing xuất khẩu đến kết quả hoạt động xuất khẩu của DN thủy hải sản của vùng $Đ B S C L$.

Trước đây, một vài nghiên cứu đã đưa các yếu tố quan hệ vào mô hình nghiên cứu để tìm kiếm và chứng minh tác động thuận chiều của yếu tố này đến kết quả hoạt động xuất khẩu, trong đó nổi bật nhất là nghiên cứu của $\mathrm{T}$. V. Nguyen và cộng sự (2018), nhóm nghiên cứu này đã đưa yếu tố quan hệ vào mô hình nghiên cứu trên cơ sở các phân tích và minh chứng của Elena (2014). Nhân tố quan hệ được xây dựng dựa trên mối quan hệ của DN trong kênh phân phối, mối quan hệ với khách hàng, với nhà cung ứng, đối tác và các tổ chức từ hiệp hội ngành nghề đến chính phủ. Tuy nhiên kết quả nghiên cứu chưa chứng minh được mối quan hệ có ý nghĩa giữa các yếu tố quan hệ với hiệu quả hoạt động xuất khẩu. Mặc dù vậy, với quan điểm các yếu tố quan hệ chính là một trong những nguồn lực hay năng lực của $\mathrm{DN}$ và hình thành sự khác biệt giữa $\mathrm{DN}$ này so với $\mathrm{DN}$ khác trên thị trường, nghiên cứu này mong muốn kiểm chứng lại một lần nữa giả thuyết H5: Các yếu tố quan hệ có tác động tích cực đến kết quả hoạt động xuất khẩu của các DN thủy hải sản vùng ĐBSCL.

O'Cass và Ngo (2011) đã đưa ra khái niệm khả năng xây dựng thương hiệu như một năng lực vững chắc của công ty. Nó bao gồm một nhóm các hoạt động như truyền thông và các chương trình tiếp thị trong việc cung cấp một ý nghĩa thương hiệu phù hợp đến với khách hàng. Merrilees, Rundle-Thiele, và Lye (2011) trong nghiên cứu của mình đã mô tả khả năng xây dựng thương hiệu bao gồm bốn cách tiếp cận: Xác định ý nghĩa thương hiệu; sử dụng thương hiệu như một công cụ hoạt động; truyền đạt ý nghĩa thương hiệu nhất quán; và nhận được sự hồ trợ thương hiệu từ nhân viên. 


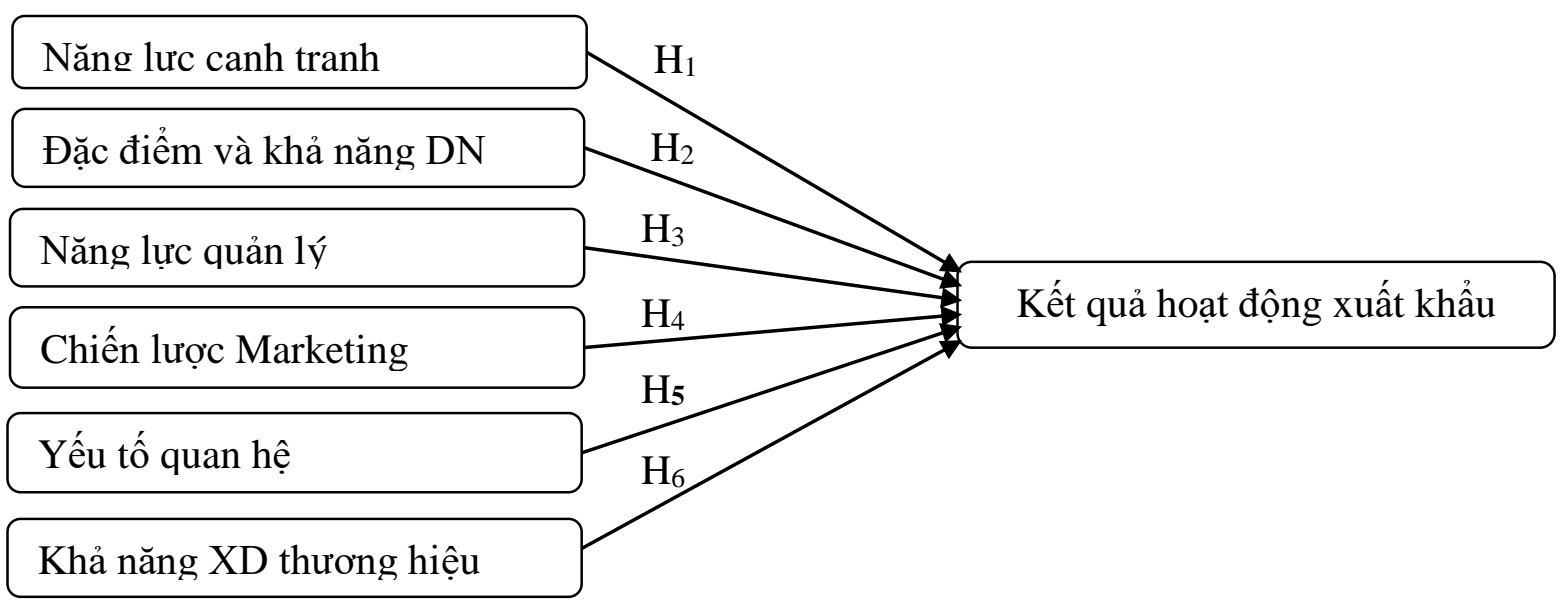

Hình 1. Mô hình nghiên cứu

Khả năng xây dựng thương hiệu khó bắt chước và chuyển giao, do đó mang lại lợi thế cạnh tranh bền vững (Morgan, Vorhies, và Mason, 2009). Xây dựng thương hiệu để bảo vệ sự đổi mới từ việc bắt chước của đối thủ cạnh tranh cũng như cho phép các công ty dễ dàng kiểm soát rủi ro và phản ứng nhanh hơn, hiệu quả hơn với những thay đổi trong thị trường (Lei, Ye, \& Abimbola, 2013). Từ nhiều bằng chứng nêu trên, nghiên cứu này kiểm chứng giả thuyết $H 6: K h a ̉$ năng xây dựng thuơng hiệu tác động tích cưc đến kết quả hoạt động xuất khẩu của DN thủy hải sản vùng ĐBSCL.

Dựa trên các giả thuyết được xây dựng, mô hình nghiên cứu được hình thành như Hình 1. Các biến trong mô hình nghiên cứu được xây dựng như trình bày trong Bảng 1 .

\section{Bảng 1}

Các biến số trong mô hình nghiên cứu

\begin{tabular}{|c|c|c|}
\hline Thành phần & Biến quan sát & Nguồn \\
\hline $\begin{array}{l}\text { Kết quả hoạt } \\
\text { động xuất } \\
\text { khẩu }\end{array}$ & $\begin{array}{l}\text { [KQHDXK1] Luôn có mật độ xuất khẩu các đơn hàng } \\
\text { dày đặt } \\
\text { [KQHDXK2] Rất hài lòng với kết quả xuất khẩu hiện tại } \\
\text { [KQHDXK3] Đã thành công với hoạt động xuất khầu } \\
\text { [KQHDXK4] Đã thâm nhập được thị trương xuất khẩu } \\
\text { [KQHDXK5] Đã đạt được sự tăng trưởng thị phần xuất } \\
\text { khẩu theo kế hoạch }\end{array}$ & $\begin{array}{l}\text { Altıntaş và cộng sự } \\
\text { (2007); V. B. Nguyen } \\
\text { và cộng sự (2017) }\end{array}$ \\
\hline $\begin{array}{l}\text { Năng lực } \\
\text { cạnh tranh }\end{array}$ & $\begin{array}{l}\text { [NLCT1] Cung cấp sản phẩm phù hợp với yêu cầu của } \\
\text { khách hàng } \\
\text { [NLCT2] Có thể cung cấp sản phẩm với chi phí thấp } \\
\text { [NLCT3] Có thể cung cấp sản phẩm với thiết kế mới nhất } \\
\text { so với đối thủ } \\
\text { [NLCT4] Có thể cung cấp các đơn hàng với số lượng lớn } \\
\text { [NLCT5] Luôn giao hàng đúng thời gian } \\
\text { [NLCT6] Linh hoạt trong việc sắp xếp các đơn hàng theo } \\
\text { đúng yêu cầu của khách hàng } \\
\text { [NLCT7] Có khả năng khai thác toàn bộ thị trường } \\
\text { [NLCT8] Có thể kiểm soát tất cả các mối đe dọa từ đối } \\
\text { thủ cạnh tranh }\end{array}$ & $\begin{array}{l}\text { Sigalas, Economou, } \\
\text { và Georgopoulos } \\
\text { (2013); } \\
\text { Siriphattrasophon và } \\
\text { Piriyatanarak (2013); } \\
\text { Kaur, Kumar, và } \\
\text { Kumar (2016); } \\
\text { Famiyeh (2017); } \\
\text { Ramlawati và } \\
\text { Kusuma (2018) }\end{array}$ \\
\hline
\end{tabular}




\begin{tabular}{|c|c|c|}
\hline Thành phần & Biến quan sát & Nguồn \\
\hline & $\begin{array}{l}\text { [NLCT9] Có khả năng mở rộng thị trường tiềm năng tốt } \\
\text { hơn so với đối thủ }\end{array}$ & \\
\hline $\begin{array}{l}\text { Đặc điểm và } \\
\text { khả năng của } \\
\text { DN }\end{array}$ & $\begin{array}{l}\text { [DD\&KNDN1] Quy mô hoạt động lớn } \\
\text { [DD\&KNDN2] Nhiều kinh nghiệm trong hoạt động xuất } \\
\text { khẩu } \\
\text { [DD\&KNDN3] Nhiều kinh nghiệm cạnh tranh trên thị } \\
\text { trường quốc tế } \\
\text { [DD\&KNDN4] Luôn xây dựng kế hoạch xuất khẩu tốt }\end{array}$ & $\begin{array}{l}\text { Nazar và Saleem } \\
\text { (2009); T. V. Nguyen } \\
\text { và cộng sự (2018) }\end{array}$ \\
\hline $\begin{array}{l}\text { Năng lực } \\
\text { quản lý của } \\
\text { DN }\end{array}$ & $\begin{array}{l}\text { [NLQL1] Ban quản lý (BQL) luôn có tầm nhìn và thái độ } \\
\text { tích cực với hoạt động xuất khẩu } \\
\text { [NLQL2] BQL có trình độ chuyên môn xuất khẩu tốt } \\
\text { [NLQL3] BQL có nhiều kinh nghiệm trong hoạt động } \\
\text { xuất khẩu } \\
\text { [NLQL4] BQL có kinh nghiệm cạnh tranh trên thị trường } \\
\text { xuất khẩu }\end{array}$ & $\begin{array}{l}\text { V. B. Nguyen và cộng } \\
\text { sự (2017) }\end{array}$ \\
\hline $\begin{array}{l}\text { Chiến lược } \\
\text { marketing } \\
\text { XK của DN }\end{array}$ & $\begin{array}{l}\text { [CLMARXK1] Áp dụng chiến lược khác biệt hóa sản } \\
\text { phẩm và đảm bảo chất lượng sản phẩm } \\
\text { [CLMARXK2] Áp dụng chương trình chiêu thị hiệu quả } \\
\text { [CLMARXK3] Thiết kế và sử dụng kênh phân phối hợp } \\
\text { lý } \\
\text { [CLMARXK4] Sử dụng chiến lược giá cạnh tranh }\end{array}$ & $\begin{array}{l}\text { V. B. Nguyen và cộng } \\
\text { sự (2017) }\end{array}$ \\
\hline $\begin{array}{l}\text { Yếu tố quan } \\
\text { hệ }\end{array}$ & $\begin{array}{l}\text { [YTQH1] Đã tạo dựng mối quan hệ phân phối chặt chẽ } \\
\text { [YTQH2] Thuộc thành viên của mạng lưới xuất khẩu, } \\
\text { hiệp hội xuất khẩu } \\
\text { [YTQH3] Đã thiết lập mối quan hệ với các tổ chức và } \\
\text { chính phủ có liên quan đến hoạt động xuất khẩu }\end{array}$ & $\begin{array}{l}\text { Elena (2014); T. V. } \\
\text { Nguyen và cộng sự } \\
\text { (2018) }\end{array}$ \\
\hline $\begin{array}{l}\text { Khả năng xây } \\
\text { dựng thương } \\
\text { hiệu }\end{array}$ & $\begin{array}{l}\text { [KNXDTH1] Sử dụng thương hiệu như một công cụ cạnh } \\
\text { tranh } \\
\text { [KNXDTH2] Có khả năng xây dựng thương hiệu có giá } \\
\text { trị } \\
\text { [KNXDTH3] Đang áp dụng cách thức xây dựng thương } \\
\text { hiệu độc đáo } \\
\text { [KNXDTH4] Đang sở hữu một thương hiệu đáng tin cậy } \\
\text { trên thị trường }\end{array}$ & $\begin{array}{l}\text { Zhang, Shen, Wu, và } \\
\text { Fan (2009); Merrilees } \\
\text { và cộng sự (2011); } \\
\text { Odoom, } \\
\text { Agbemabiese, } \\
\text { Anning-Dorson, và } \\
\text { Mensah (2017) }\end{array}$ \\
\hline
\end{tabular}

Nguồn: Tổng hợp

\section{Dữ liệu và phương pháp nghiên cứu}

Theo Hair, Anderson, Tatham, và Black (1998) để cỡ mẫu đảm bảo phù hợp với phương pháp phân tích EFA thì số quan sát ít nhất phải bằng 4-5 lần số biến, trong nghiên cứu này có 33 biến quan sát tức cần từ 132 đến 165 quan sát. Vì vậy, nghiên cứu tiến hành điều tra và thu được 170 quan sát. Đối tượng khảo sát là các DN có hoạt động xuất khẩu thủy hải sản tại các tỉnh thành thuộc vùng ĐBSCL. Vì tính chất khó tiếp cận của $\mathrm{DN}$ cũng như yêu cầu đối tượng trả lời phỏng vấn phải thuộc cấp lãnh đạo của $\mathrm{DN}$ nên nghiên cứu đã sử dụng phương pháp thuận tiện để khảo sát.

Dựa trên lược khảo tài liệu, bộ tiêu chí đo lường các nhân tố ảnh hưởng đến kết quả hoạt động xuất khẩu đã được xây dựng và điều chỉnh phù hợp phạm vi và đặc trưng ngành 
nghề. Thang đo Likert 5 mức độ được sử dụng để đánh giá với 1 là "hoàn toàn không đồng ý” cho đến 5 là "hoàn toàn đồng ý". Nghiên cứu sử dụng Cronbach's Alpha để kiểm định thang đo trước khi áp dụng hai phương pháp định lượng là phân tích nhân tố khám phá và ước lượng mô hình hồi qui tuyến tính. Thực hiện phân tích nhân tố khám phá để rút gọn các biến tạo ra các biến độc lập và biến phụ thuộc cho phân tích hồi qui. Mục tiêu của phân tích là xây dựng một hàm tuyến tính để cho thấy sự tác động cũng như mức độ tác động của các nhân tố được nghiên cứu đến kết quả hoạt động xuất khẩu của DN.

Mô hình hồi qui có dạng:

$$
\mathrm{Y}_{\mathrm{i}}=\mathrm{B}_{0}+\mathrm{B}_{1} \mathrm{X}_{1 \mathrm{i}}+\mathrm{B}_{2} \mathrm{X}_{2 \mathrm{i}}+\ldots+\mathrm{B}_{\mathrm{k}} \mathrm{X}_{\mathrm{ki}}+\mathrm{e}_{\mathrm{i}}
$$

Trong đó:

Y: Kết quả hoạt động xuất khẩu

X1: Năng lưc cạnh tranh; X2: Đặc điểm và khả năng của DN; X3: Năng lục quản lý; X4:Chiến luợc Marketing xuất khẩu; X5: Yếu tố quan hệ; X6: Khả năng xây dựng thuơng hiệu.

\section{Kết quả phân tích và thảo luận}

Trước khi tiến hành phân tích nhân tố khám phá, nghiên cứu sử dụng phương pháp kiểm định thống kê bằng hệ số Cronbach's Alpha để kiểm tra mức độ chặt chẽ của các mục hỏi trong bộ tiêu chí đo lường năng lực cạnh tranh. Kết quả phân tích ở Bảng 2 cho thấy, các biến đo lường tốt cho từng thang đo thành phần sau khi loại một số biến như KQHDXK1, NLCT1, $D D \& K N D N 1$, và $C L M A R X K 3$ khỏi thang đo. Tiến hành phân tích nhân tố, kết quả có 6 nhân tố được rút ra cho nhóm các biến độc lập, giải thích được $62,74 \%$ biến thiên của dữ liệu điều tra. Tương tự như vậy cho biến phụ thuộc, kết quả cho thấy các đo lường trong biến phụ thuộc sau khi gom nhóm giải thích được 58,12\% biến thiên (Bảng 3 ).

\section{Bảng 2}

Kết quả độ tin cậy của thang đo

\begin{tabular}{llc}
\hline \multicolumn{1}{c}{ Nhóm thành phần } & \multicolumn{1}{c}{ Biến } & Cronbach's Alpha \\
\hline Kết quả hoạt động xuất khẩu & KQHDXK1, KQHDXK2, & 0,736 \\
& KQHDXK3, KQHDXK4, & \\
& KQHDXK5 & \\
Năng lự cạnh tranh & NLCT1, NLCT2, NLCT3, NLCT4, & 0,894 \\
& NLCT5, NLCT6, NLCT7, NLCT8, & \\
& NLCT9 & 0,716 \\
Đặc điểm và khả năng của DN & DD\&KNDN1, DD\&KNDN2, \\
& DD\&KNDN3, DD\&KNDN4 & \\
Năng lực quản lý & NNQL1, NNQL2, NNQL3, NNQL4 & 0,736 \\
Chiến luợc marketing XK & CLMARXK1, CLMARXK2, & 0,751 \\
Yếu tố quan hệ & CLMARXK3, CLMARXK4 \\
Khả năng xây dựng thuơng hiệu & YTQH1, YTQH2, YTQH3 & 0,621 \\
& KNXDTH1, KNXDTH2, KNXDTH3, & 0,719 \\
\hline
\end{tabular}

Nguồn: số liệu khảo sát 170 DN thủy hải sản vùng ĐBSCL, 2019 


\section{Bảng 3}

Kết quả phân tích ma trận nhân tố sau khi xoay

\begin{tabular}{lr}
\hline Biến & Hệ số tải nhân tố \\
\hline KQHDXK2 & 0,787 \\
KQHDXK3 & 0,882 \\
KQHDXK4 & 0,750 \\
KQHDXK5 & 0,605 \\
\hline KMO: 0,712; P value: 0,000; \\
Phurong sai trich: $58,12 \%$ \\
\hline
\end{tabular}

\begin{tabular}{lc}
\hline Biến & Hệ số tải nhân tố \\
\hline NLCT2 & $\mathbf{0 , 6 9 7}$ \\
NLCT3 & $\mathbf{0 , 7 3 3}$ \\
NLCT4 & $\mathbf{0 , 7 5 4}$ \\
NLCT5 & $\mathbf{0 , 7 8 5}$ \\
NLCT6 & $\mathbf{0 , 7 7 5}$ \\
NLCT7 & $\mathbf{0 , 7 6 8}$ \\
NLCT8 & $\mathbf{0 , 6 9 9}$ \\
NLCT9 & $\mathbf{0 , 7 8 9}$ \\
\hline DD\&KNDN3 & 0,767 \\
DD\&KNDN4 & 0,683 \\
\hline NLQL1 & $\mathbf{0 , 5 7 7}$ \\
NLQL2 & $\mathbf{0 , 7 7 3}$ \\
NLQL3 & $\mathbf{0 , 5 8 1}$ \\
NLQL4 & $\mathbf{0 , 7 0 8}$ \\
\hline CL MARXK1 & 0,811 \\
CL MARXK2 & 0,838 \\
CL MARXK4 & 0,544 \\
\hline YTQH1 & $\mathbf{0 , 8 4 4}$ \\
YTQH2 & $\mathbf{0 , 5 9 6}$ \\
YTQH3 & $\mathbf{0 , 7 4 7}$ \\
\hline DD\&KNDN2 & 0,512 \\
KNXDTH1 & 0,633 \\
KNXDTH3 & 0,541 \\
KNXDTH4 & 0,772 \\
\hline KMO: 0,$810 ;$ P value: 0,$000 ;$ \\
Phuơng sai trich: $62,74 \%$ \\
\hline
\end{tabular}

Nguồn: Số liệu khảo sát 170 DN thủy hải sản vùng ĐBSCL, 2019

Kết quả phân tích ở Bảng 3 cho thấy, các kiểm định được đảm bảo như sau: (1) Kiểm định tính thích hợp của mô hình $(0,5<\mathrm{KMO}=0,712$ và $0,810<1,0)$ tương ứng biến phụ thuộc và biến độc lập; (2) Kiểm định Bartlett's về sự tương quan của các biến quan sát (Sig. $=0,000$ $<0,05)$ cho cả hai nhóm biến chứng tỏ các biến có liên quan chặt chẽ với nhau; Hệ số tải nhân tố của các biến đều lớn hơn 0,5 (Hair, Black, Babin, \& Anderson, 2010). Điều này cho thấy, kết quả phân tích EFA là phù hợp.

Các nhóm nhân tố được rút trích và gom nhóm lại với một số khác biệt nhỏ không đáng kể so với các nhóm biến xây dựng ban đầu. Kết quả này cho thấy mô hình nghiên cứu đề xuất lúc này đã có sự điều chỉnh nhưng về số biến và tên gọi các biến độc lập vẫn không thay đổi so với mô hình nghiên cứu đề xuất. Giá trị Sig. của kiểm định $\mathrm{F}$ là 0,000 ; chứng tỏ giả thuyết $\mathrm{H} 0$ đã bị bác bỏ và tồn tại mối quan hệ tuyến tính giữa biến phụ thuộc $\mathrm{Y}$ với ít nhất một biến trong các biến độc lập đưa vào mô hình. Hệ số $\mathrm{R}^{2}=0,359$ tức mô hình giải thích được $35,9 \%$ sự biến thiên của Y (kết quả hoạt động xuất khẩu) bởi mối quan hệ tuyến tính của các nhân tố tác động (Bảng 4). Nghiên cứu cũng tiến hành một số kiểm định như phân phối chuẩn và giả định về hiện tượng đa cộng tuyến và tự tương quan, tất cả đều đạt yêu cầu. Cụ thể, dựa vào hệ số phóng đại phương sai VIF của các biến có thể thấy không có hiện tượng đa cộng tuyến xảy ra vì VIF 
của các biến đều bé hơn 10 (Mai, 2006). Kết quả hệ số Durbin Watson của mô hình $\mathrm{d}=1,678$ tiến tới giá trị 2 . Do đó, hiện tượng tự tương quan chuỗi bậc nhất không ảnh hưởng đến ý nghĩa của mô hình hồi qui (Hair et al., 2010).

Kết quả phân tích ở Bảng 4 cho thấy, mô hình hồi qui có 4 biến có ý nghĩa thống kê tác động đến kết quả hoạt động xuất khẩu của các $\mathrm{DN}$, bao gồm: Năng lực cạnh tranh, Đặc điểm và khả năng của DN, Yếu tố quan hệ và Khả năng xây dựng thương hiệu. Giá trị Beta chuẩn hóa của 4 biến này cho thấy các biến độc lập đều có mối quan hệ thuận chiều đến kết quả hoạt động xuất khẩu. Trong đó, nhân tố khả năng xây dựng thưong hiệu có tác động mạnh nhất $(0,329)$. Điều này phù hợp với thực tế hoạt động xuất khẩu thủy hải sản của vùng ĐBSCL khi mà các $\mathrm{DN}$ cạnh tranh với nhau ngày càng gay gắt hơn thì yếu tố niềm tin vào thương hiệu của từng doanh nghiệp phải được chú trọng để phân biệt $\mathrm{DN}$ này với $\mathrm{DN}$ khác trong bối cảnh điều kiện sản xuất nuôi trồng tương tự nhau như hiện nay. Hơn nữa, các DN này chủ yếu chế biến và xuất khẩu là tôm, cá và mực đông lạnh sang các thị trường như Mỹ, châu Âu, Nhật Bản... với các yêu cầu từ thị trường ngày càng khắt khe mà đặc biệt là về nguồn nguyên liệu đầu vào. Cho nên, một $\mathrm{DN}$ nào xây dựng được niềm tin về sự ổn định của chất lượng sản phẩm thể hiện bằng thương hiệu của mình thì hiệu suất xuất khẩu của $\mathrm{DN}$ đó càng cao. Kết luận này cũng phù hợp với nghiên cứu của Lei và cộng sự (2013) khi chứng minh được việc xây dựng thương hiệu vững chắc sẽ giúp $\mathrm{DN}$ phản ứng hiệu quả hơn đối với những biến động của thị trường từ đó nâng cao kết quả hoạt động xuất khẩu.

\section{Bảng 4}

Mức độ ảnh hưởng của các nhân tố đến kết quả hoạt động xuất khẩu của các DN

\begin{tabular}{|c|c|c|c|c|c|c|}
\hline \multirow[b]{2}{*}{ Mô hình } & \multirow{2}{*}{$\begin{array}{c}\text { B chưa } \\
\text { chuẩn } \\
\text { hóa }\end{array}$} & \multirow{2}{*}{$\begin{array}{l}\text { Sai số } \\
\text { chuẩn }\end{array}$} & \multirow{2}{*}{$\begin{array}{c}\text { Beta } \\
\text { chuẩn } \\
\text { hóa }\end{array}$} & \multicolumn{2}{|c|}{ Mức ý nghĩa } & \multirow{2}{*}{$\begin{array}{c}\text { Hệ số } \\
\text { phóng đại } \\
\text { phương } \\
\text { sai (VIF) }\end{array}$} \\
\hline & & & & $\mathbf{t}$ & Sig. & \\
\hline Hà̀ng số & $-5,359^{* * * *}$ & 0,594 & & $-9,025$ & 0,000 & \\
\hline Năng lục cạnh tranh & $0,186^{* *}$ & 0,078 & 0,154 & 2,385 & 0,018 & 1,065 \\
\hline Đăc điềm và $K N D N$ & $0,258^{* *}$ & 0,109 & 0,188 & 2,371 & 0,019 & 1,604 \\
\hline Năng lực quản lý & $0,187^{n s}$ & 0,120 & 0,118 & 1,558 & 0,121 & 1,454 \\
\hline Chiến luợc Marketing XK & $0,022^{n s}$ & 0,120 & 0,014 & 0,184 & 0,854 & 1,455 \\
\hline Yếu tố quan hệ & $0,206 * *$ & 0,089 & $\mathbf{0 , 1 5 3}$ & 2,297 & 0,023 & 1,124 \\
\hline Khả năng xây dưng TH & $0,590 * * *$ & 0,138 & 0,329 & 4,265 & 0,000 & 1,512 \\
\hline \multicolumn{7}{|c|}{ Biến phu thuộc: Kết quả hoạt động xuất khẩu (KQHDXK) } \\
\hline \multicolumn{7}{|l|}{ R: 0,$600 ;$ R Square: 0,359} \\
\hline \multicolumn{7}{|l|}{ Durbin-Watson: 1,678} \\
\hline$F: 15,244 ; P$ value: 0,000 & & & & & & \\
\hline
\end{tabular}

*: ý nghĩa thống kê 10\%, **: ý nghĩa thống kê 5\%, ***: ý nghĩa thống kê 1\%, ns: không có ý nghĩa thống kê

Nguồn: Số liệu khảo sát 170 DN thủy hải sản vùng ĐBSCL, 2019

Nhân tố đặc điểm và khả năng của $D N$ có giá trị hệ số Beta đứng thứ hai về mức độ tác động $(0,188)$ và cũng tác động cùng chiều đến kết quả hoạt động xuất khẩu. Kết quả chứng minh được rằng $\mathrm{DN}$ với càng nhiều kinh nghiệm trên thị trường quốc tế và hoạch định càng tốt kế hoạch xuất khẩu thì hiển nhiên sẽ tăng hiệu quả hoạt động xuất khẩu. Môi trường cạnh tranh trên thị trường xuất khẩu thủy hải sản thay đổi nhanh và liên tục theo tình hình thế giới cho nên kinh nghiệm ở đây không đi liền với số năm thành lập của $\mathrm{DN}$ mà phải đồng hành với kinh nghiệm cạnh tranh và việc $\mathrm{am}$ hiểu sâu sắc về hoạt động xuất khẩu. Bản thân nghiên cứu của 
T. V. Nguyen và cộng sự (2018) đã chứng minh thuyết phục về sự tác động của nhân tố này đến hoạt động xuất khẩu của DN trong chính ngành hải sản tại Việt Nam.

Số liệu Bảng 4 cũng cho thấy không có sự khác biệt quá lớn giữa vị trí tác động thứ ba và thứ tư đến kết quả hoạt động xuất khẩu. Cả nhân tố năng lực cạnh tranh và yếu tố quan hệ có mức độ tác động gần như nhau tương ứng 0,154 và 0,153 . Kết quả này đã cùng lúc củng cố nghiên cứu của Han và cộng sự (2007) và Famiyeh (2017) vì họ tin rằng có mối quan hệ nhân quả giữa năng lục cạnh tranh và kết quả hoạt động xuất khẩu trong đó năng lực cạnh tranh thay đổi sẽ thúc đẩy kết quả hoạt động xuất khẩu cũng thay đổi cùng chiều. Lẽ đương nhiên khi các DN xuất khẩu thủy hải sản trang bị cho mình năng lực cạnh tranh càng vượt trội hơn so với đối thủ thì khả năng cung cấp đa dạng và kiểm soát tốt thị trường cũng như phản ứng kịp thời và nhanh chóng với các thay đổi từ thị trường thì sẽ dẫn đến một kết quả tất yếu là nâng cao được kết quả hoạt động xuất khẩu. Về các yếu tố quan hệ, nếu nghiên cứu của $\mathrm{T}$. V. Nguyen và cộng sự (2018) chưa tìm ra được minh chứng cho sự tác động tích cực từ các yếu tố quan hệ đến hiệu quả hoạt động xuất khẩu thì nghiên cứu này đã chứng minh được điều đó. Đây cũng là kết quả ít được chứng minh trong các nghiên cứu trước đây, đặc biệt trong nhóm ngành xuất khẩu thủy hải sản tại Việt Nam. Về thực tế thì tất cả mọi DN đều hiểu rằng khi $\mathrm{DN}$ xây dựng được mối quan hệ bao gồm quan hệ với đối tác, khách hàng và trung gian càng chặt chẽ thì khả năng phản ứng và đối phó lại các biến động của thị trường càng cao làm cho kết quả hoạt động xuất khẩu luôn được đảm bảo.

Các nhân tố như năng lưc quản lý và chiến lược marketing xuất khẩu, mặc dù được kỳ vọng sẽ tác động rất lớn đến kết quả hoạt động xuất khẩu, nhưng chưa được chứng minh có tác động có ý nghĩa thống kê đến kết quả hoạt động xuất khẩu của DN. Điều này đi ngược lại với kết quả của V. B. Nguyen và cộng sự (2017). Nguyên nhân của vấn đề này có thể xuất phát từ phương pháp chọn mẫu là thuận tiện nên đa số các doanh nghiệp đồng ý trả lời khảo sát là các doanh nghiệp có quy mô nhỏ và vừa. Theo đặc thù của các doanh nghiệp nhỏ và vừa ở ĐBSCL thì phần lớn họ quản lý theo kinh nghiệm và theo hình thức $\mathrm{DN}$ gia đình nên bản thân cũng chưa chú trọng thay đổi nhiều trong công tác quản lý hay xây dựng đội ngũ quản lý có năng lực. Cũng bởi cách thức hoạt động của nhiều $\mathrm{DN}$ xuất khẩu thủy hải sản vùng ĐBSCL chủ yếu theo kinh nghiệm và dựa trên các mối quan hệ đối tác xây dựng lâu dài từ trước nên việc hình thành các chiến lược marketing xuất khẩu như các hoạt động nghiên cứu thị trường, tổ chức thiết kế, sản xuất và cung ứng ra thị trường chưa được quan tâm áp dụng. Đa phần các $\mathrm{DN}$ này sẽ sản xuất theo đặt hàng từ đối tác sẵn có. Kết quả này cũng dễ dàng nhận thấy đó là các sản phẩm mà các $\mathrm{DN}$ vùng ĐBSCL đã xuất khẩu thời gian qua chủ yếu là sản phẩm thô mà không phải các sản phẩm giá trị gia tăng.

\section{Kết luận và khuyến nghị cho doanh nghiệp}

Kết quả nghiên cứu trên cho thấy bốn nhân tố quan trọng tác động cùng chiều đến kết quả hoạt động xuất khẩu của DN thủy hải sản vùng ĐBSCL là khả năng xây dựng thưong hiệu, đặc điểm và khả năng của DN, năng lục cạnh tranh và yếu tố quan hệ.

Dựa trên kết quả phân tích hồi qui, nghiên cứu đề xuất bốn khuyến nghị góp phần nâng cao kết quả hoạt động xuất khẩu của DN như sau:

Một là, tập trung xây dựng thương hiệu và áp dụng các qui trình hiện đại vào trong quá trình xây dựng thương hiệu cho DN thông qua xây dựng bộ nhận diện thương hiệu, chỉ dẫn địa lý, trong đó chú trọng đặc biệt đến việc duy trì chất lượng sản phẩm đúng cam kết với nhà nhập 
khẩu để nuôi dưỡng giá trị thương hiệu trong dài hạn. Các DN nên sử dụng thương hiệu như một công cụ cạnh tranh trên thị trường và tích cực phát huy lợi thế của thương hiệu. Việc xây dựng thương hiệu trong bối cảnh ngày nay đòi hỏi phải có sự chuyên nghiệp cao, do đó các DN nên thuê chuyên gia về thương hiệu tham gia tư vấn và thực hiện quá trình xây dựng thương hiệu để không phải đối mặt những tình trạng như hiện nay là thương hiệu được xây dựng không đến nơi đến chốn, làm hạn chế hiệu quả của thương hiệu mang lại cho DN. Các DN cần nhận thức đầy đủ và đúng hơn về vai trò của thương hiệu trong hoạt động xuất khẩu hiện nay.

Hai là, tích lũy thêm kinh nghiệm xuất khẩu thông qua hình thức xuất khẩu trực tiếp thay vì việc lựa chọn xuất khẩu gián tiếp hay ủy thác như hiện nay. Việc xuất khẩu trực tiếp sẽ tạo cơ hội cho $\mathrm{DN}$ hình thành và xây dựng được cho mình một thương hiệu có giá trị, qua đó góp phần vào thực hiện việc xây dựng thương hiệu hiệu quả. Thêm đó, $\mathrm{DN}$ phải luôn chuẩn bị sẵn sàng nhiều phương án hay kế hoạch xuất khẩu để tăng khả năng linh hoạt và chủ động trong việc ứng phó các thay đổi từ thị trường.

Ba là, chú trọng nâng cao năng lực cạnh tranh bắt đầu bằng việc đầu tư nâng cao thiết kế sản phẩm mới với chi phí cạnh tranh so với đối thủ và đảm bảo các đơn hàng với số lượng lớn cũng như đảm bảo thời gian giao hàng đúng yêu cầu của khách hàng. Muốn thực hiện tốt điều này, các $\mathrm{DN}$ nên chú trọng nhiều hơn nữa việc lập và thực hiện chiến lược marketing xuất khẩu, loại bỏ tư tưởng làm theo thói quen, theo kinh nghiệm, theo cái mình có mà phải dựa trên nhu cầu thị trường. Một chiến lược marketing xuất khẩu hiệu quả sẽ giúp DN có cơ sở vững chắc để phản ứng linh hoạt với các mối đe dạo từ thị trường và từ đối thủ.

Cuối cùng, $\mathrm{DN}$ phải chú trọng vào các yếu tố quan hệ. Việc xây dựng và củng cố các mối quan hệ nên được tập trung đầu tiên vào khách hàng thông qua việc vận dụng các công cụ của quản trị quan hệ khách hàng $(\mathrm{CRM})$ hiệu quả. Luôn chú trọng vào việc tìm kiếm để thiết lập, duy trì và mở rộng quan hệ với khách hàng. $\mathrm{DN}$ cần xây dựng mối quan hệ với nhà cung ứng để thiết lập một chuỗi cung ứng đảm bảo cả về chất lượng và số lượng. Chất lượng cung ứng sẽ giúp DN duy trì được giá trị thương hiệu của mình. Số lượng cung ứng sẽ giúp nâng cao năng lực cạnh tranh trên qui mô để đáp ứng các đơn hàng của nhà nhập khẩu. Ngoài ra, cũng phải thực hiện xây dựng quan hệ với các trung gian phân phối trong kênh phân phối, một mối quan hệ bền vững trong kênh sẽ giúp DN có thể lắng nghe nhu cầu thị trường, giảm áp lực chi phí trung gian và đảm bảo sự chắc chắn trong đầu ra của sản phẩm. Thêm vào đó, $\mathrm{DN}$ nên tích cực tham gia vào hiệp hội và các tổ chức có liên quan, thiết lập các kênh thông tin để tăng cường trao đổi và nắm bắt các thông tin hữu ích từ hiệp hội cũng như chính phủ.

\section{Các hạn chế của đề tài và hướng nghiên cứu tiếp theo}

Nghiên cứu tồn tại một số hạn chế cần được khắc phục trong những nghiên cứu tiếp theo. Một là việc sử dụng mô hình hồi quy tuyến tính là chưa đủ mạnh để chứng minh mối quan hệ trung gian của các biến như năng lực cạnh tranh và chiến lược marketing xuất khẩu trong mô hình này. Đề xuất các nghiên cứu sau nên sử dụng phương pháp SEM để tìm hiểu sâu hơn các mối quan hệ trung gian trong mô hình. Hai là, cỡ mẫu khảo sát được của nghiên cứu là chưa đủ lớn để thể hiện tính bao quát cho vùng nghiên cứu. Các nghiên cứu tiếp theo nên tăng cỡ 
mẫu và mở rộng các vùng nghiên cứu khác nhau để có cái nhìn toàn diện hơn về vấn đề nghiên cứu. Việc tăng cỡ mẫu sẽ thích hợp hơn cho việc áp dụng phương pháp SEM vào mô hình nghiên cứu. Ba là phương pháp chọn mẫu của nghiên cứu là phương pháp thuận tiện nên cũng mang lại nhiều hạn chế về độ biến động của dữ liệu và độ tin cậy của nghiên cứu. Các nghiên cứu tiếp theo nên sử dụng phương pháp chọn mẫu ngẫu nhiên phân tầng để làm tăng độ tin cậy cho nghiên cứu.

\section{Tài liệu tham khảo}

Adu-Gyamfi, N., \& Korneliussen, T. (2013). Antecedents of export performance: The case of an emerging market. International Journal of Emerging Markets, 8(4), 354-372.

Agus, A. A., Isa, M., Farid, M. F., \& Permono, S. P. (2015). An assessment of SME competitiveness in Indonesia. Journal of Competitiveness, 7(2), 60-74.

Altıntaş, M. H., Tokol, T., \& Harcar, T. (2007). The effects of export barriers on perceived export performance: An empirical research on SMEs in Turkey. EuroMed Journal of Business, 2(1), 36-56. doi:10.1108/14502190710749947

Arafah, W., Batara, R. R., \& Hady, H. (2018). Antecedents and consequence of entrepreneurial strategic orientation in micro business unit in Jakarta. International Journal of Entrepreneurship, 22(2), 1-17.

Chen, J., Sousa, C. M. P., \& He, X. (2016). The determinants of export performance: A review of the literature 2006-2014. International Marketing Review, 33(5), 626-670. doi:10.1108/imr-10-2015-0212

Elena, B. S. (2014). Determinants and measures of export performance-comprehensive literature review. Journal of Contemporary Economic and Business Issues, 1(1), 63-74.

Erdil, T. S., \& Ozdemir, O. (2016). The determinants of relationship between marketing mix strategy and drivers of export performance in foreign markets: An application on Turkish clothing industry. Procedia - Social and Behavioral Sciences, 235, 546-556.

Famiyeh, S. (2017). Corporate social responsibility and firm's performance: Empirical evidence. Social Responsibility Journal, 13(2), 390-406. doi:10.1108/SRJ-04-2016-0049

Guan, J. C., Yam, R. C. M., Mok, C. K., \& Ma, N. (2006). A study of the relationship between competitiveness and technological innovation capability based on DEA models. European Journal of Operational Research, 170(3), 971-986. doi:10.1016/j.ejor.2004.07.054

Hair, J. F, Anderson, R. E., Tatham, R. L., \& Black, W. C. (1998). Multivariate data analysis with readings (5th ed.). Upper Saddle River, NJ: PrenticeHall.

Hair, J. F., Black, W. C., Babin, B. J., \& Anderson, R. E. (2010). Multivariate data analysis: A global perspective (7th ed.). Upper Saddle River, NJ: Pearson Prentice Hall.

Han, S. B., Chen, S. K., \& Ebrahimpour, M. (2007). The impact of ISO 9000 on TQM and business performance. The Journal of Business and Economic Studies, 13(2), 1-11. 
Karim, M. A., Smith, A. J. R., Halgamuge, S. K., \& Islam, M. M. (2008). A comparative study of manufacturing practices and performance variables. International Journal of Production Economics, 112(2), 841-859. doi:10.1016/j.ijpe.2007.07.005

Katsikeas, C. S., Leonidou, L. C., \& Morgan, N. A. (2000). Firm-level export performance assessment: Review, evaluation, and development. Journal of the Academy of Marketing Science, 28(4), 493-511.

Kaur, S. P., Kumar, J., \& Kumar, R. (2016). Impact of flexibility of manufacturing system components on competitiveness of SMEs in Northern India. Journal of Engineering, Project \& Production Management, 6(1), 63-76. doi:10.32738/JEPPM.201601.0006

Lei, X., Ye, T., \& Abimbola, T. (2013). The role of branding capability for innovative companies: Stock market reactions to new product announcement. Nankai Business Review International, 4(4), 329-348.

Mai, V. N. (2006). Giáo trình kinh tế luợng [The econometrics curriculum]. Hanoi, Vietnam: Nhà xuất bản thống kê.

Merrilees, B., Rundle-Thiele, S., \& Lye, A. (2011). Marketing capabilities: Antecedents and implications for B2B SME performance. Industrial Marketing Management, 40(3), 368375. doi:10.1016/j.indmarman.2010.08.005

Moghaddam, F. M., Hamid, A. B. B. A., \& Aliakbar, E. (2012). Management influence on the export performance of firms: A review of the empirical literature 1989-2009. African Journal of Business Management, 6(15), 5150-5158. doi:10.5897/AJBM11.1408

Morgan, R. E., Vorhies, D. W., \& Mason, C. H. (2009). Market orientation, marketing capabilities, and firm performance. Strategic Management Journal, 30(8), 909-920.

Navarro, A., Losada, F., Ruzo, E., \& Diez, J. A. (2010). Implications of perceived competitive advantages, adaptation of marketing tactics and export commitment on export performance. Journal of World Business, 45, 49-58. doi:10.1016/j.jwb.2009.04.004

Nazar, M. S., \& Saleem, H. M. N. (2009). Firm level determinants of export performance. International Business \& Economics Research Journal, 8(2), 105-112. doi:10.19030/iber.v8i2.3107

Nguyen, T. V., Phuoc, M. H., Nguyen, T. L., Phan, M. Đ., \& Vo, K. N. (2018). The empirical study on seafood export performance in the Mekong Delta, Vietnam. Academy of Marketing Studies Journal, 22(2), 131-145.

Nguyen, V. B., Le, T. B., Nguyen, T. V., \& Vo, K. N. (2017). Determinants of export performance: Case of seafood firms in Viet Nam. Business and Economic Horizons (BEH), 13(5), 722-735. doi:10.15208/beh.2017.48

O’Cass, A., \& Ngo, L. V. (2011). Achieving customer satisfaction in services firms via branding capability and customer empowerment. Journal of Services Marketing, 25(7), 489-496. doi:10.1108/08876041111173615

Odoom, R., Agbemabiese, G. C., Anning-Dorson, T., \& Mensah, P. (2017). Branding capabilities and SME performance in an emerging market: The moderating effect of brand regulations. Marketing Intelligence \& Planning, 35(4), 473-487. doi:10.1108/MIP08-2016-0138 
Phan, V. H. (2009). Nuôi trồng thủy sản Thùa Thiên Huế trong bối cảnh tụ do hóa thuoong mại [Aquaculture in Thua Thien Hue in the context of trade liberalization]. (Doctoral dissertation, Hue University, Vietnam). Retrieved March, 12, 2019, from http://luanan.nlv.gov.vn/luanan?a=d\&d=TTbGWOSzvnau2009

Phòng Thương mại và Công nghiệp Việt Nam. (2017). Báo cáo Chỉ số Năng lục cạnh tranh cấp tỉnh của Việt Nam tì̀ năm 2011 - 2016 [Report on Vietnam's Provincial Competitiveness Index from 2011 - 2016]. Hanoi, Vietnam: Phòng Thương mại và Công nghiệp Việt Nam.

Porter, M. E. (2008). On competition, updated and expanded edition. Boston, MA: Harvard Business School Press.

Ramlawati, R., \& Kusuma, A. H. P. (2018). Total quality management as the key of the company to gain the competitiveness, performance achievement and consumer satisfaction. International Review of Management and Marketing, 8(5), 60-69.

Rojoka, J. (2009). Baltic states competitiveness: Before and after the global crisis. Applied Economics: Systamatic Research, 3(1), 27-46.

Salloum, M. (2013). Explaining the evolution of performance measures - A dual case-study approach. Journal of Engineering, Project, and Production Management, 3(2), 99-106.

Sanchez, R., \& Heene, A. (1996). A systems view of the firm in competence-based competition. In Dynamics of competence-based competition (pp. 39-62). Oxford, UK: Pergamon.

Sigalas, C., Economou, V. P., \& Georgopoulos, N. B. (2013). Developing a measure of competitive advantage. Journal of Strategy and Management, 6(4), 320-342.

Siriphattrasophon, S., \& Piriyatanarak, S. (2013). A causal model of ethical business practices and firm competitiveness of small and medium enterprises in Thailand. International Journal of Arts \& Sciences, 6(2), 389-402.

Xuan Thao (2017). Thách thức lớn đối với mục tiêu xuất khẩu tôm đạt 10 tỷ USD [The big challenge for shrimp export target of 10 billion USD]. Retrieved May 19, 2017, from http://doanhnhan.net/thach-thuc-lon-doi-voimuc-tieu-xuat-khau-tom-dat-10-ty-usd113770.html

Zhang, X., Shen, L., Wu, Y., \& Fan, L. C. (2009). Competitiveness assessment for real estate enterprises in China: A model of procedure. International Journal of Strategic Property Management, 13(3), 229-245. 